\title{
LA INICIATIVA DE INTEGRACIÓN DEL PLAN PUEBLA PANAMÁ: CONSIDERACIONES DESDE LA POSTURA DEL DESARROLLO REGIONAL EQUILIBRADO DE MESOAMÉRICA
}

Crucita Aurora Ken Rodríguez, Universidad de Quintana Roo, Departamento de Ciencias Económico Administrativo de la

División de Ciencias Sociales y Económico Administrativa, Boulevard Bahía Chetumal 77000, Quintana Roo . Tel: (983) 8350300

Correo electrónico: cruken@uqroo.mx

\section{RESUMEN}

Las integraciones regionales que la globalización empuja a emprender son asumidas por los países como signo de inserción a la corriente actual del crecimiento. En su afán de ampliar sus áreas de influencia, los países desarrollados promueven, globalmente, las prácticas liberales del comercio y las inversiones en los países menos desarrollados a través de estas integraciones. El Plan Puebla Panamá (PPP) es una de estas iniciativas de integración que ha gozado de aceptación y de promoción de parte de los países desarrollados y de los gobiernos de los países centroamericanos, de México y Columbia. Esta región, hoy llamado "Mesoamérica" alberga a una importante población urbana y rural, donde esta último se caracteriza por ser indígena y campesina. Las necesidades de desarrollo de esta población son de naturaleza económica, social y ambiental, mientras que el PPP enfatiza lo comercial, financiero y la privatización. Por consiguiente, este ensayo presenta un análisis de las bases principales del PPP, plantea una crítica desde la óptica del desarrollo regional equilibrado, formula algunas propuestas y finalmente, expone una evaluación sobre la viabilidad de reformular el PPP para convertirlo en un mecanismo de desarrollo regional equilibrado en Mesoamérica.

Palabras clave: Desarrollo regional, Desarrollo local, Gestión pública, Desarrollo sustentable, Papel del Estado, Equilibrio regional , Participación ciudadana. 
THE INTEGRATION INITIATIVE OF PLAN PUEBLA

PANAMA : CONSIDERATIONS FROM THE BALANCING REGIONAL DEVELOPMENT OF MESIAMERICA

Crucita Aurora Ken Rodríguez, Universidad de Quintana Roo, Departamento de Ciencias Económico Administrativo de la

División de Ciencias Sociales y Económico Administrativa , Boulevard Bahía Chetumal 77000, Quintana Roo . Tel: (983) 8350300

Correo electrónico: cruken@uqroo.mx

\section{ABSTRACT}

Regional integrations are promoted by the globalization process and are viewed by many countries as a sign of growth. Developed countries benefit from the formation of regional blocks in less developed countries by expanding their global markets through the promotion of free trade and investments. One such integration is the Plan Puebla Panamá (PPP) that has received the endorsement of developed countries and the consent of the Central American governments, Mexico and Colombia. This region, commonly called today "Mesoamerica" is inhabited by an important population living in both the urban and rural areas, in the later composed of a significant number of farmers and indigenous people. The necessity of bringing economic, social and environmental development to this region is paramount. However, the PPP emphasizes commercial and financial growth and privatization. Consequently, this essay is an analysis of the basis principles of the PPP from a regional development perspective, it presents proposals and finally it evaluates the viability of making the PPP a mechanism for the equitable regional development of Mesoamerica

Keywords: Regional development, local development, Public Management, Sustainable Development, Role of State, Regional Balance, Citizen Participation. 


\section{LAS POLÍTICAS Y ESTRATEGIAS PRINCIPALES DEL PLAN PUEBLA PANAMÁ}

El Plan Puebla Panamá es una de las iniciativas de integración más recientes en la región centroamericana. Fue inicialmente promovido por el gobierno de México con apoyo del Banco Interamericano de Desarrollo (BID) en septiembre del 2000 y decretado en el 2001 bajo la presidencia de Vicente Fox. Se promueve esta iniciativa de integración basada en el desarrollo desequilibrado de México, donde la región sur sureste se reconoce como la más marginada (Dávila, Kessel y Levy, 2000) lo que sirvió de base para promover la vinculación de esta región de México con los países centroamericanos que también sufren de niveles similares de marginación.

Tabla No.1

\begin{tabular}{|l|c|c|c|}
\hline \multicolumn{4}{|c|}{ Subregión México } \\
\hline Estado & $\begin{array}{c}\text { Índice de margi- } \\
\text { nación }\end{array}$ & $\begin{array}{c}\text { Grado de margi- } \\
\text { nación }\end{array}$ & Lugar \\
\hline Guerrero & 2.412 & Muy alto & 1 \\
\hline Chiapas & 2.326 & Muy alto & 2 \\
\hline Oaxaca & 2.129 & Muy alto & 3 \\
\hline Veracruz & 1.076 & Alto & 4 \\
\hline Puebla & 0.634 & Alto & 7 \\
\hline Campeche & 0.558 & Alto & 8 \\
\hline Tabasco & 0.462 & Alto & 9 \\
\hline Yucatán & 0.431 & Alto & 11 \\
\hline Quintana Roo & -0.315 & Bajo & 19 \\
\hline
\end{tabular}

Indicadores de marginación de los estados integrantes del Plan Puebla Panamá a 2005 Fuente: Consejo Nacional de Población, México, 2005.

Se observa que los cuatro primeros lugares de marginación en México lo ocupan estados que pertenecen a la región sur sureste. Es importante mencionar que según un análisis en el 2010 realizado por el gobierno mexicano señala que si bien el índice nacional de pobreza multidimensional extrema es $10.5 \%$, en los grupos indígenas llega a 39\% (UNDP, 2011:85). Esto nos indica la situación de mayor 
pobreza entre la población indígena en México.

Por consiguiente, el PPP incluyó a los estados mexicanos de Puebla, Oaxaca, Guerrero, Veracruz, Tabasco, Chiapas, Campeche, Yucatán y Quintana Roo, y siete naciones centroamericanas: Belice, Guatemala, Honduras, El Salvador, Nicaragua, Costa Rica y Panamá. Sin embargo, el 11 de julio de 2006, Colombia se convirtió en miembro pleno. El plan se planteó para una duración de 25 años.

En junio de 2001, México jurídicamente establece la Coordinación General del PPP como una instancia de coordinación cuyo objeto principal es el de impulsar el desarrollo integral sustentable de la región sur-sureste del país y promover, a través de los conductos oficiales, la participación de los países de la región de Centroamérica a fin de instrumentar acciones conjuntas que contribuyan al logro del desarrollo integral de dicha región, con apego al respeto de la soberanía de las naciones y a los principios de derecho internacional (Gobierno de los Estados Unidos Mexicanos, 2001: 1-2). En el PPP se propone la realización de consulta continua y permanente para establecer la estrategia regional integral y de largo plazo, a través de la participación de los actores claves de las comunidades, pueblos, organizaciones ciudadanas, empresarios y los distintos órdenes de gobierno para garantizar el respeto a la libertad de las regiones y entidades en la definición de su propio destino (Gobierno de los Estados Unidos Mexicanos, 2000: 3).

El apoyo del BID fue un elemento importante para instituir el PPP pues aportó certeza a la obtención de créditos para los proyectos propuestos a implementarse en la región. Las iniciativas del PPP a propuesta del BID fueron definidas como las siguientes: 1. Desarrollo sustentable; 2. Desarrollo humano; 3. Prevención y mitigación de desastres naturales; 4. Promoción al turismo; 5 . Facilitación del intercambio comercial; 6 . Integración vial; 7. Interconexión energética; y 8. Integración de servicios de telecomunicación.

\subsection{Creación y modernización de Infraestructura}

En primer lugar, el PPP trata de propiciar la construcción de infraestructura en la región para atraer inversiones y facilitar el comercio, posicionando a los países miembros en la competitividad mundial del intercambio. Para el PPP la infraestructura vial es de suma importancia para facilita el comercio entre Mesoamérica y sus socios comerciales importantes: Estados Unidos, China, y otros países desarrollados. Las conexiones viales con los puertos y terminales y la red de carreteras se hace necesaria para aumentar igualmente la actividad turística. 
Por esta razón los proyectos propuestos se localizan en polos turísticos y maquiladores dejando excluidas áreas como Belice que no son importantes en esas actividades. Actualmente, se han aprobado proyectos de ampliación de carreteras fronterizas, ampliación de infraestructura portuaria y aduanal, expansión y mejoras de aeropuertos y la modernización de vías ferroviarias. Para ello, el BID, la Comisión Económica para América Latina (CEPAL), el Banco Centroamericano de Integración Económica (BCIE) y otros bancos, se han enfocado al desarrollo de nuevos ejes de infraestructura interoceánica principalmente en la actual región del Canal de Panamá, en Honduras y en el istmo de Tehuantepec (Barreda, 2002).

La Iniciativa de Integración Energética, que incluye la interconexión eléctrica y gasífera, constituye otra de las grandes líneas de acción del PPP. En este marco, se ha venido trabajando en la estructuración del Sistema de Interconexión Eléctrica Centroamericana (SIEPAC), que apunta al objetivo de unificar e interconectar los mercados eléctricos de la región, con miras a promover el aumento de las inversiones privadas en el sector y una reducción del precio de la electricidad. Este proyecto, ha sido retomado por el Programa de Integración Energética Mesoamericano (PIEM), adoptado en la Cumbre de Cancún, en diciembre de 2005 por parte de los países miembros del Sistema de Integración Latinoamericano (SICA), a saber, Colombia, México y República Dominicana. EI PIEM es parte de una estrategia encaminada a la adopción de soluciones que permitan afrontar la crisis energética de los países centroamericanos, originada por el alza del petróleo y su impacto en las economías locales, buscando la conformación de un mercado de hidrocarburos con cobertura regional.

Los proyectos de interconexión eléctrica y gasífera entre Colombia y Panamá, han constituido un punto de partida para el desarrollo de las propuestas en el escenario del PPP y del PIEM dando relevancia a la participación del país, y permitiendo concebir una propuesta de integración, aprovechando la posición estratégica de Colombia como país de enlace entre Centroamérica y la Región Andina, en la perspectiva de conformar un mercado eléctrico supraregional. Lo anterior se ha visto reforzado con los avances que se registran en el Gasoducto Transcaribeño, tramo Antonio Ricaurte, entre Colombia y Venezuela, y la propuesta de articularlo a Panamá, como paso previo a la conformación de un mercado con los países centroamericanos. 


\subsection{Avances de los proyectos}

Durante el período 2002 - 2006, se registraron avances significativos en 33 proyectos regionales, que involucran recursos del orden de 4,500 millones de dólares, destacando los siguientes: (i) avances para la operación del Mercado Eléctrico Regional Centroamericano (MER); (ii) inicio de la construcción de las líneas de transmisión eléctrica Guatemala - México; (iii) elaboración de estudios de preinversión para la línea de interconexión eléctrica entre Panamá y Colombia; (iv) creación del Programa de Integración Energética Mesoamericana (PIEM); (v) puesta en ejecución de programas de electrificación rural que beneficiarán a más de 300,000 nuevos usuarios en Guatemala y Honduras; (vi) avances en la promoción del uso de energías renovables mediante estudios del potencial geotérmico y el impulso de proyectos que promuevan el uso de fuentes hídrica, eólica y solar; (vii) avances sustanciales en la ejecución de los planes de inversión para el mejoramiento, rehabilitación, ampliación y señalización de cerca de 4,000 kilómetros de tramos carreteros incluidos en la Red Internacional de Carreteras Mesoamericanas (RICAM); (viii) conclusión de los estudios de factibilidad técnicafinanciera de la red de fibra óptica que conformará la Autopista Mesoamericana de la Información (AMI); (ix) avances para la definición de una estrategia de impulso a la Sociedad de la Información con apoyo de la CEPAL; $(x)$ inicio de la ejecución de programas sociales como el Programa Mesoamericano de Vigilancia Epidemiológica, el Programa Mesoamericano de Abordaje del VIH SIDA; (xi) inicio del Programa de Desarrollo Sostenible de Comunidades Rurales en Áreas Naturales Multinacionales; y (xii) el inicio de la preparación del Atlas Centroamericano de Información Territorial para el Desarrollo y la Reducción de Riesgo a Desastres.

\subsection{Promoción del desarrollo socioeconómico}

En la actualidad la región PPP se constituye de la siguiente población: en la región sur sureste de México viven 31.7 millones de habitantes, que representan más de uno de cada cuatro mexicanos (el $28.2 \%$ de la población total del país).

En el año 2010 el 42.6\% de los habitantes de la región mexicana del PPP residía en dos de los estados de ella, Puebla y Veracruz. Destaca este último como el más poblado (con casi 8 millones de personas). Por otra parte, Campeche es la entidad menos poblada con apenas 0.8 millones de habitantes, población inferior incluso que la de Quintana Roo o Tabasco.

En cuanto a los otros países integrantes del PPP el más poblado es Colombia con el 
$38.6 \%$ de la población total de la región, seguido por México con el $26.3 \%$, luego Guatemala que duplica el número de habitantes del próximo que es Honduras. En orden descendente le sigue El Salvador, Nicaragua, Costa Rica, Panamá y Belice, que cuenta con una población mínima, incluso inferior al del estado de Campeche en México. Es observable que en cuanto a población, la región del PPP presenta desigualdades significativas. Teóricamente, el PPP es una estrategia regional para potencializar el desarrollo económico, reducir la pobreza y acrecentar la riqueza del capital humano y el capital natural de la región mesoamericana dentro de un contexto de respeto a la diversidad cultural y étnica, y con la inclusión de la sociedad civil.

Tabla No.2

\begin{tabular}{|c|c|c|c|}
\hline \multicolumn{2}{|c|}{ Subregión México } & \multicolumn{2}{|c|}{ Subregión Centro América } \\
\hline Puebla & 5.8 & Belice & 0.3 \\
\hline Oaxaca & 3.8 & Guatemala & 14.4 \\
\hline Guerrero & 3.4 & Honduras & 7.6 \\
\hline Veracruz & 7.7 & San Salvador & 6.2 \\
\hline Tabasco & 2.2 & Nicaragua & 5.8 \\
\hline Chiapas & 4.8 & Costa Rica & 4.6 \\
\hline Campeche & 0.8 & Panamá & 3.5 \\
\hline Quintana Roo & 1.3 & Colombia & 46.3 \\
\hline Yucatán & 1.9 & \multirow[b]{2}{*}{ Total } & \multirow[b]{2}{*}{88.7} \\
\hline Total & 31.7 & & \\
\hline
\end{tabular}

Distribución poblacional de los estados y países integrantes del Plan Puebla Panamá, en millones, 2012.

Fuente: Instituto Nacional de Estadística y Geografía, Censo de Población y Vivienda, México, 2012 y United Nation

Lo que concierne a los habitantes de la región, su situación se atiende a través de la Iniciativa de Desarrollo Humano del PPP, la cual está encaminada a reducir la pobreza, facilitar el acceso a los servicios sociales básicos de la población 
vulnerable y contribuir al pleno desarrollo de los pueblos mesoamericanos. Es importante mencionar que la región del PPP comprende una significante porción de población indígena: $48 \%$ de la población total de Guatemala (CEPAL, 2000), 33\% en Yucatán, 32.6\% en Oaxaca, y el $20 \%$ en Chiapas y Quintana Roo (INEGI, 2000) que viven en las zonas rurales, practican la agricultura y tiene fuertes costumbres comunitarias.

Tabla No.3

\begin{tabular}{|l|c|c|c|c|}
\hline \multirow{2}{*}{$\begin{array}{l}\text { Países Inte- } \\
\text { grantes }\end{array}$} & \multicolumn{2}{|l|}{$\begin{array}{l}\text { Índices de Desarrollo Hu- } \\
\text { mano }\end{array}$} & $\begin{array}{c}\text { Esperan- } \\
\text { za de vida } \\
\text { al nacer }\end{array}$ & $\begin{array}{c}\text { Ingreso Nacio- } \\
\text { nal Bruto per } \\
\text { cápita } \\
\text { (PPP US \$ de }\end{array}$ \\
\cline { 2 - 3 } & 2000 & 0.725 & 79.1 & 10,870 \\
\hline Costa Rica & 0.684 & 0.755 & 76.0 & 13,347 \\
\hline Panamá & 0.703 & 0.689 & 73.4 & 8,589 \\
\hline Colombia & 0.637 & 0.750 & 76.7 & 13,971 \\
\hline México & 0.698 & 0.659 & 72.0 & 6,498 \\
\hline El Salvador & 0.606 & 0.604 & 72.6 & 3,750 \\
\hline Honduras & 0.552 & 0.565 & 73.8 & 2,567 \\
\hline Nicaragua & 0.512 & 0.560 & 70.8 & 4,694 \\
\hline Guatemala & 0.514 & 0.694 & 76.9 & 5,693 \\
\hline Belice & ------ & & & \\
\hline
\end{tabular}

Índice de Desarrollo Humano y otros indicadores Socioeconómicos de los países integrantes del Plan Puebla Panamá, 2010.

Fuente: United Nation $\$$

Actualmente, los países integrantes del PPP con los índices humanos más bajos son Honduras, Nicaragua y Guatemala. Asimismo, Guatemala, El Salvador, Honduras, Nicaragua y Columbia muestran los más bajos niveles de esperanza de vida, mientras que Nicaragua, Honduras, Belice y el Salvador registran ingresos nacional bruto per cápita más bajos. Sin embargo, Panamá, Costa Rica y México son los países que reportan los mejores niveles en los indicadores de desarrollo humano. Esto demuestra que existe desequilibrio en los niveles de bienestar en la región 
que integra el PPP.

Para atender la parte económica del PPP se crea el Consejo Mesoamericano de Competitividad (CMC) cuyo objetivo fundamental es la formulación de una Agenda Mesoamericana de Competitividad que dirija en una forma estratégica acciones encaminadas a mejorar la competitividad por medio del aumento de la productividad empresarial y el fomento de las inversiones. Bajo este marco se propuso articular los esfuerzos de infraestructura, cadenas productivas, clima de negocios, promoción de inversiones, comercio regional, desarrollo del recurso humano e innovación tecnológica.

Otros temas de importancia económica es la Iniciativa de Promoción del Turismo orientada a promover el desarrollo del turismo ecológico, cultural e histórico, mediante acciones regionales que destacaran la complementariedad, las economías de escala y los encadenamientos productivos del turismo. De igual modo se creó la Iniciativa de Facilitación del Intercambio Comercial, la cual propone el fomento del intercambio en la región mediante una reducción de los costos de transacción, y la promoción de las pequeñas y medianas empresas en las exportaciones. Es importante mencionar que el Consejo Mesoamericano de Competitividad fue creado por la Iniciativa de Facilitación del Intercambio Comercial.

\subsection{El PPP y el desarrollo sustentable}

La región sur sureste de México es muy rica en recursos naturales. Tiene una gran variedad de suelos y una biodiversidad importante que representa casi el $70 \%$ de la biodiversidad total del país. Oaxaca, Chiapas, Veracruz y Guerrero son los cuatro estados con mayor riqueza de especies animales y vegetales, y Puebla ocupa el sexto lugar. Casi la mitad de la fauna protegida por la legislación mexicana y los tratados internacionales, y el $60 \%$ de la flora endémica y protegida se localizan en esta región.

Estos estados también cuentan con agua en abundancia y ríos significativos; concentra los más grandes acuíferos del país y los menos explotados. Esta región incluye precisamente a los estados de México que reciben los mayores volúmenes de precipitación media anual, mismos que están muy por encima de la media nacional $(772 \mathrm{~mm})$. La gran disponibilidad de agua en la región es sin duda uno de los grandes activos de la misma. Además goza de una extensión costera amplia, tiene extensiones importantes de bosques y selvas, con importantes yacimientos 
de hidrocarburos y minerales metálicos y no metálicos, y alberga un número importante de reservas naturales.

Respecto a los recursos forestales, destaca el hecho de que las únicas entidades en México cuya cobertura de bosques es superior al $50 \%$ de sus respectivos territorios se encuentran en esta región; se trata de Quintana Roo, Campeche, Guerrero, Oaxaca, y Chiapas, cuya cobertura está por encima de la media nacional. De igual modo, en la región sur sureste del país se encuentran los remanentes de selva tropical Iluviosa (Gobierno de los Estados Unidos Mexicanos, 2000: 56-59).

Sin embargo, según el documento base del PPP, capítulo México, la biodiversidad de la región sur sureste de este país se encuentra amenazada por las poblaciones rurales de menores recursos, que sin otros medios de producción a su alcance, hacen uso intensivo de los recursos naturales que conforman su hábitat. Los altos grados de contaminación en parte de las cuencas de la región, y el sistema de roza -tumba-quema, que, además de presentar grave peligro de provocar incendios forestales no controlados, requiere la apertura anual de un promedio de 4 hectáreas de arbolado por jefe de familia. "Por lo anterior, considerando la riqueza de recursos naturales y la baja calidad ecológica de algunos estados de la región sur sureste, es necesario advertir los principales riesgos de deterioro ambiental en la región que podrían inhibir el desarrollo sustentable esperado. Aunado a que las actividades productivas de los habitantes de la región sur sureste constituyen un riesgo ambiental, pudiendo provocar una extinción masiva y selectiva de especies animales y vegetales" (Gobierno de los Estados Unidos Mexicanos, 2000: 53). Sin embargo, es importante mencionar que la población rural campesina e indígena son propietarios de estos recursos naturales importantes para la biodiversidad.

El área de la biodiversidad del PPP se atiende a través de la Iniciativa de Desarrollo Sustentable, en la cual se promueve la conservación y el manejo sustentable de los recursos naturales y los mecanismos participativos, especialmente de las comunidades locales, en la gestión ambiental. En este marco se han identificado los siguientes proyectos: gestión ambiental; patrimonio cultural y equidad; preservación y aprovechamiento de los recursos naturales, y el fondo mesoamericano para el desarrollo. 


\section{EL PLAN PUEBLA PANAMÁ DESDE LA ÓPTICA DEL DESARROLLO REGIONAL}

El PPP es una iniciativa de integración que se emprende con el propósito de aliviar la marginación de los países de Mesoamérica. Desde la visión del desarrollo regional, las integraciones son agrupaciones de naciones que aun siendo distintas, buscan un avance común a través del aprovechamiento de sus riquezas. Sin embargo, estas agrupaciones económicas crean un mapa de la nueva repartición de los espacios entre las potencias económicas en búsqueda de la ampliación de los mercados para el creciente comercio. Según Tamames (1991: 192), "es preciso realizar una serie de actuaciones de acoplamiento de las estructuras nacionales, a fin de llegar con el mínimo costo social en el ámbito que se pretende integrar", consejo que, en lo general, no se ha acatado en el proceso de integración regional del PPP.

Dentro del enfoque de desarrollo regional, la búsqueda del desarrollo comunitario se ha traducido en el paradigma de desarrollo local y endógeno, paradigma que incorpora la participación de la ciudadanía, el equilibrio en el desarrollo y el papel de un Estado innovador, democrático, transparente y con rendición de cuentas. Lo anterior nos lleva a requerir una gestión pública acorde con las necesidades del desarrollo endógeno para promover el desarrollo regional que buscan estas integraciones. La necesidad de una nueva gestión pública ha conducido a nuevas prácticas y a la acuñación de términos que transforman la práctica monopólica de la administración pública en procesos de autogestión, cogestión, contraloría social y presupuesto participativo, entre otros.

En los países subdesarrollados, el proceso de globalización lleva a la necesidad de la integración, por lo que las políticas públicas toman un enfoque más tendiente a modelos exógenos que se expresan en políticas que se alejan de los intereses y beneficios de las poblaciones locales. En el PPP, el enfoque del desarrollo es exógeno, modelo que ha hecho evidente el ensanche de la brecha entre ricos y pobres, entre industrializados y no industrializados. De la promoción de las políticas públicas exógenas en la región de Mesoamérica se han encargado las agencias y bancos internacionales como la Agencia Estadounidense de Ayuda Internacional para el Desarrollo (USAID), el BID y el BM, afiliadas en apoyo al PPP. Sin embargo, las políticas públicas endógenas sufren de orfandad por la falta de promoción de parte de las agencias internaciones, de la falta de interés de los gobiernos locales y de la falta de organizaciones ciudadanas con fuerza suficiente para lograr incluir sus necesidades de desarrollo en las agendas públicas. 
Actualmente, la población del mundo manifiesta su escepticismo sobre las ventajas sociales, culturales, políticas y ambientales provenientes de las integraciones. Los beneficios y ventajas económicas comerciales de las integraciones son los motores más potentes para proponerlas y son las más anunciadas y convincentes para aceptarlas. Sin embargo, con la importancia que ha tomado el crecimiento económico como medio para aliviar la pobreza, las integraciones se proponen y se implementan empujando a la gente a la modernización, aludiendo al supuesto sinónimo entre crecimiento y desarrollo, entre modernización y desarrollo, entre libre comercio y desarrollo, entre inversión privada extranjera y desarrollo, semejanzas forzadas que muchos autores no ortodoxos de los años 50, los estructuralistas latinoamericanos y algunos actuales han comprobado engañosos.

Las integraciones con objetivos comerciales y de inversiones refuerzan la importancia del papel de la sociedad civil, las ONG y del Estado en el diseño del desarrollo con ese ingrediente fundamental de la participación ciudadana. Esta simbiosis de Estado y ciudadanía requiere de reestructuraciones institucionales y administrativas hacia la Nueva Gestión Pública (NGP) a nivel local y regional. Esta NGP por naturaleza se constituye en un modelo endógeno de administración del Estado que conlleva impresa la responsabilidad hacia el desarrollo de los pueblos y de la región, responsabilidad que se traduce en saber conjugar el modelo de desarrollo endógeno (desarrollo desde dentro) con el modelo exógeno que presiona por espacios de poder económico y político extranacional.

El desarrollo regional equilibrado se manifiesta cuando cada región de un territorio goza un nivel de vida digna como fruto de los beneficios socioeconómicos derivados del aprovechamiento de sus potencialidades y capacidades endógenas. Por ejemplo, (i) producir y vender a un mercado justo sus productos que siempre han producido bajo pocos apoyos efectivos; (ii) recibir apoyo para mejorar los servicios de salud, educación, vivienda e infraestructura social y productiva que impacte en su calidad de vida; (iii) mejorar sus ingresos a través de aumentos en la productividad y mejores condiciones de mercado con capacitaciones y apoyo técnico específico; (iv) mejorar la comercialización por medio de cadenas productivas inter y entre regiones; $(v)$ recibir el apoyo y aval del gobierno en el diseño de políticas públicas, proyectos y programas según las necesidades de cada región; y (vi) beneficiarse de un gobierno que busca crear un entorno favorable para el desarrollo rural y urbano propiciando formas nuevas de interacción entre los sectores sociales, privados y públicos. Desde mi óptica de desarrollo regional, el PPP, si bien favorece la instalación de una infraestructura moderna que servirá 
para atraer la inversión extranjera, deja en exclusión a un segmento importante de la economía campesina e indígena cuyos problemas de producción, ingresos y servicios sociales en las áreas rurales no son abordados de manera explícita en las discusiones de infraestructura e inversiones.

\subsection{La nueva gestión pública y el desarrollo regional}

Según Guerrero (2003: 86), en la NGP "los servicios públicos se consideraron a la luz de preocupaciones sociales y políticos entonces vigentes, observándose que la administración pública debería tener la capacidad de resolver problemas relativos a la población, la industrialización, los alimentos, el medio ambiente, la urbanización y el desarrollo rural; problemas específicos y en momentos concretos a manera de hechura de policy". Para el PPP, la gestión pública debe responder a un diseño autóctono regional, es decir, diseñada desde las características internas, propias, únicas del espacio que lo va a ejercer, para que los servicios que rinda sean acorde a los esperados por sus habitantes para frenar las desigualdades, la marginación, el deterioro cultural y ambiental y la explotación y aprovechamiento exógeno de sus riquezas.

En mi opinión, el PPP enfrenta este reto, pero los gobiernos aún no están conscientes de ello pues solamente ven y responden a las presiones del modelo exógeno promovido por la globalización y operacionalizado por las integraciones regionales. EI PPP abarca una población importante indígena, con sus lenguas en peligro de desaparición, población mayormente agropecuaria con fuertes componentes comunitarios y que históricamente ha sido saqueado por modelos económicos exógenos de "crecimiento hacia fuera", situación señalada por los estructuralistas latinoamericanos que también exigieron un vuelco a un modelo de "desarrollo hacia dentro". Por lo anterior, para que esta región se detone se requiere de la participación de la ciudadanía y del Estado para planear el desarrollo endógeno y así potencializar las productividades y las riquezas locales de los habitantes para hallar el camino hacia un desarrollo regional equilibrado en Mesoamérica.

Las políticas públicas que proponen beneficiar a sus habitantes conllevan el sello endógeno. Es decir que incorporan en su formulación las aspiraciones de la gente de llevar una vida digna y de gozar de libertades como por ejemplo de continuar siendo campesinos, artesanos, de vivir en la zona rural o urbana, de acceder a la educación, a la alimentación, a la vivienda digna, a la salud. Las políticas públicas 
van de la mano con la planeación y con el papel del Estado. Para enfocarse al desarrollo endógeno y regional, el Estado se tiene que asumir como un actor clave endógeno a la región mesoamericana.

La oposición al PPP surge principalmente por sus fundamentos basados en el modelo exógeno de diseño neoliberal ignorando el modelo endógeno que comprende las fuertes culturas y tradiciones de gran parte de la población mesoamericana, ligadas a las tierras y a sus vidas comunales. En América Latina y el Caribe se han realizado investigaciones que exploran el acceso a la tierra. Este aspecto de la discriminación demuestra que concentrarse en el crecimiento económico general puede beneficiar a los pueblos indígenas, pero es improbable que sea suficiente para cerrar la brecha. Se necesitan estrategias focalizadas, según las propuestas de los propios pueblos indígenas, y sustentadas en sus opiniones y prioridades (UNDP, 2011: 85), o sea, planear el desarrollo con procesos participativos.

Esta situación detectada por algunas organizaciones civiles en toda la región llevó a movimientos que hicieron disminuir la promoción del PPP como proyecto integrador. Sin embargo, aunque se piensa que el PPP ha quedado estancado, los proyectos de infraestructura carretera, hidroeléctrica y de comunicaciones siguen su marcha bajo las políticas públicas de los países de manera individual, que reciben créditos de diversos bancos de desarrollo para llevar a cabo las obras públicas para agilizar el comercio en la región, que al concluir, completarían la red de transporte y comunicaciones trazado desde el principio, y alcanzarán los objetivos plasmados del PPP.

En este sentido, es importante confrontar dos conceptualizaciones sobre el desarrollo. Por un lado, Jeffrey Sachs (2006) pertenece al grupo de economistas que piensa y promueve la igualdad entre maquiladoras y desarrollo, mientras que, por otro lado, Amartya Sen (2000) quien cuestiona la manera usual de los países desarrollados de promover el crecimiento económico y la "modernización" contra los intereses de la gente, vistos desde lo que estas imposiciones representan en la expansión de las libertades para salir de la pobreza, pues las decisión que se toman y las políticas públicas que se formulan no incorporan la participación de la sociedad.

Este enfoque fundamental de las políticas públicas endógenas se adapta al PPP con muchos beneficios para la población, dando como resultado políticas públicas regionales para el desarrollo equilibrado de Mesoamérica. Pero tanto a nivel micro como suprarregional, se requiere de cambios en la educación, capacitación, en la 
ideología y en los instrumentos jurídicos incluyendo la Constitución, para dar cabida a nuevas prácticas democráticas a fin de alcanzar el desarrollo como un Derecho humano.

\section{Tabla No.4}

Indicadores de la educación de los países integrantes del Plan Puebla Panamá, 2012.

\begin{tabular}{|l|c|c|}
\hline \multicolumn{1}{|c|}{ Países Integrantes } & $\begin{array}{c}\text { Años } \\
\text { promedios de }\end{array}$ & $\begin{array}{c}\text { \% de alfabeti- } \\
\text { zación }\end{array}$ \\
\hline Costa Rica & 8.3 & 96.0 \\
\hline Panamá & 9.4 & 93.5 \\
\hline Colombia & 7.4 & 93.4 \\
\hline México & 8.7 & 92.9 \\
\hline El Salvador & 7.7 & 84.0 \\
\hline Honduras & 6.5 & 83.6 \\
\hline Nicaragua & 5.7 & 78.0 \\
\hline Guatemala & 4.1 & 73.8 \\
\hline Belice & 9.2 & ----- \\
\hline
\end{tabular}

Fuente: United Nation 20 Development Program, 2011.

En ese sentido vemos que actualmente los países que integran el PPP muestran diferentes niveles de educación tanto desde los años promedio de instrucción como por el porcentaje de alfabetización. Podemos notar que Panamá y Belice son los más altos, seguido por México y Costa Rica, mientras que Nicaragua y Guatemala tienen los niveles más bajos en años de escolaridad y en porcentaje de alfabetización. Concuerdo con Santana (1994: 205) de que los espacios locales brindan unas determinadas condiciones favorables a estos procesos de modernización democrática, pero para que ellos sean posibles y reales se requiere que diversos sectores sociales asuman el proceso de democratización política y social en cada localidad en concreto. $Y$ para lo anterior, la educación juega un papel importante. 


\subsection{El papel del Estado en el desarrollo regional}

Según Sen (2000) la legitimidad está íntimamente ligada a las políticas públicas y a las acciones del Estado, y contienen una expresión más elevada a los derechos humanos. En este sentido, el desarrollo humano, el desarrollo endógeno y el desarrollo regional son parte determinantes de los derechos humanos. El desarrollo como derecho humano finca una obligación al Estado como garante de su consecución. En cuestión de los elementos que causan la pobreza como la falta de educación, salud, alimentación, vivienda y seguridad económica, la obligación de proveerlo es del Estado, auxiliado por las políticas públicas endógenas, formuladas para potencializar las capacidades de su población de manera equitativa en las regiones. Chang (1996) nos recuerda la posición estratégica del Estado para representar el interés de sus habitantes, situación que hoy se enfatiza con la creciente consciencia de que la gente constituye la riqueza de las naciones (UNDP, 1990, 2011).

Considero que desde el punto de vista del desarrollo regional, la gestión pública implica la participación del Estado como responsable de involucrar a la ciudadanía en la planeación del desarrollo, y que para las iniciativas de integración regional es necesario ampliar la participación de los ciudadanos de los países integrantes, transparentar el proceso y rendir cuentas sobre los avances y sus impactos en materia económica, social, política y ambiental. El Estado, desde el punto de vista de Chang (1996:183) "es el único organismo que puede representar el interés de la sociedad en conjunto, si bien resultaría ingenuo dar por sentado que todos los Estados que existen tienen la coherencia organizativa, el deseo político o el poder para explotar esa posición estratégica en beneficio nacional". Sin embargo, siendo el Estado una institución endógena, desde la posición del desarrollo regional, es el garante del desarrollo de sus habitantes a los cuales representa en la toma de decisiones sobre los beneficios y las condiciones de la integración. La integración como vía para el desarrollo es favorable cuando las comunidades están en condiciones de ampliar sus potencialidades endógenas para gozar de mayor libertad en el sentido que Sen (2000) propone.

Por ello, sostengo que el papel del Estado frente a las propuestas de integración es también de informar a sus habitantes, ejerciendo la transparencia, para que utilicen su libertad política de discutir y decidir las condiciones de la integración. En la perspectiva de NGP el papel del Estado es, una vez decidida la integración, rendir cuentas a sus habitantes sobre los procesos y los resultados, para que se hagan las provisiones necesarias en cuestión de políticas públicas a fin de evitar 
externalidades negativas o impactos sociales, económicos, políticos o ambientales desfavorables. Las integraciones benéficas, que impactan positivamente en el desarrollo endógeno de las comunidades y en el desarrollo equilibrado de la región están íntimamente relacionadas a la nueva gestión pública que exige un papel del Estado con principios democráticos que establece la búsqueda del bienestar con la práctica responsable de la transparencia, la rendición de cuentas y la participación ciudadana.

\subsection{El desarrollo regional equilibrado}

Los habitantes requieren que sus gobiernos que avalan y promueven las integraciones aseguren que éstas les permitan ampliar sus posibilidades de mejorar su calidad de vida en un ambiente de armonía con sus hermanos vecinos, lo que equivale a decir que la integración propicie el desarrollo regional de los países involucrados de manera equilibrada. Desde esta óptica, considero que para garantizar las ventajas que prometen las integraciones, la gente necesariamente debe estar incluida en el diseño de políticas públicas, las que deben ser conducentes a potencializar los recursos endógenos para dar cabida a los avances y beneficios de la integración, en una perspectiva de desarrollo regional equilibrado.

Las estrategias para un desarrollo equilibrado tienen que ser expresadas as nivel local enfocados al desarrollo endógeno, orientados a la integralidad de las actividades a nivel nacional preservando y mejorando el nivel sociocultural. El desarrollo que se propone en el PPP trae como resultado el incremento del comercio mundial en la región mesoamericana; la infiltración de empresas transnacionales enormemente potentes en comunicación y en carretera (Associació d'Amistat amb el Poble de Guatemala, 2004: 22 y 23); el aumento de la especulación financiera y de capital productivo (Delgado, 2004); la renuncia del Estado del bienestar dejando en manos privadas la electricidad, telefonía, salud, educación, la alimentación; la entrega de tecnología propia a patentes extranjeros para luego pagar por su utilización; estructuras militares que vigilan el complimiento de políticas; y la emigración interna e internacional por el abandono de las políticas públicas en apoyo a la población campesina y por la poca importancia otorgada al desarrollo endógeno en general.

Consecuentemente, de no asegurar el desarrollo endógeno y regional equilibrado, las integraciones deben ser vistas con mucha cautela porque más que emisarias de 
usos y costumbres de los pueblos y de la causante de mayor desigualdad y desequilibrio. Considero que la gente como riqueza verdadera de toda nación, reconociéndose como tal o actuando por intuición, saben que deben ser incluidos en el diseño de planes de integración y en la formulación de las políticas públicas para asegurar que respondan a las nuevas amenazas que vulneran su bienestar y su libertad. La gente, como el centro del desarrollo, exige políticas públicas que garanticen la libertad política, el empoderamiento, la sustentabilidad y la seguridad humana, porque el desarrollo es hoy un derecho humano que debe ser asegurado por los gobiernos.

\subsection{La participación ciudadana para el desarrollo regional}

Hoy se considera que el desarrollo se tiene que manifestar a nivel de comunidades para asegurar que el impacto sea directo y eficiente. Sin embargo, el problema ha radicado en que el donador o diseñador de los proyectos de desarrollo parte de una definición a priori y sin suficiente conocimiento de las comunidades que pretende desarrollar, de lo que significa el desarrollo para ellos, y de cómo requieren que se exprese. En muchos casos la "modernización" que conlleva etiquetado las integraciones regionales significa la migración campo ciudad por la

E\&A IIES agudización de la pobreza de campesinos y la transculturación forzada de los habitantes comunitarios, porque en lugar de apoyarlos a modernizar el campo y las zonas rurales potencializando sus capacidades endógenas, promueven proyectos urbanos generadores de empleos de baja remuneración.

EI PPP como proyecto de integración para el desarrollo regional sufre de la misma debilidad de los proyectos de desarrollo aplicados antes de los años setentas. Lo que es lo mismo decir que el PPP no satisface las necesidades de las comunidades locales. Por ejemplo, la red vial prioriza las supercarreteras y no la comunicación carretera a nivel local que ayudaría a conectar a las comunidades de la zona rural con mercados locales. De inicio, el PPP no fue precedido por un diagnóstico de capacidades endógenas, por lo que no ofrece oportunidades de potencializar los recursos endógenos para el desarrollo local de las comunidades de los países que lo integran.

EI PPP busca la modernización rural a través de la urbanización de los campesinos, que en el pensar de Jeffery Sachs (2006) sería positivo, aunque esto implique la pérdida de cultura y biodiversidad, pérdida de tierras, lenguas, relaciones comunitarias, conocimientos ancestrales, usos y costumbres, y la pérdida de 
solidaridad y cohesión comunitaria. Otros proyectos públicos y privados se están llevando a cabo y se planean sin tomar en cuenta las violaciones del derecho indígena y campesino a la tierra, lo que va en contra de acuerdos internacionales incluyendo el Convenio 169 de la Organización Internacional del Trabajo sobre los derechos de las poblaciones indígenas a mantener sus costumbres y sus tierras. Los proyectos que buscan establecer los inversionistas en esta región, como por ejemplo las hidroeléctricas, son causantes de afectación ambiental y humana. Estos proyectos también son implementados sin los estudios de impactos ambientales, sociales y culturales correspondientes.

\section{EL PLAN PUEBLA PANAMÁ DESDE LA ÓPTICA DEL DESARROLLO REGIONAL}

Como hemos enfatizado, el desarrollo que propone el PPP es acorde a la política neoliberal que favorece la ampliación del capital de las empresas extranjeras, mientras que el desarrollo endógeno dirige los beneficios hacia la población local dentro de la misma iniciativa de integración. El siguiente cuadro nos contrasta esas dos opciones, en donde el enfoque regional equilibrado exige nuevas bases y lineamientos para un desarrollo humano acorde a las aspiraciones de la población de Mesoamérica. Las propuestas parten de las críticas hechas por las poblaciones afectadas que han encontrado eco en las organizaciones sociales y asociaciones civiles que promueven cambios del PPP.

\section{Tabla No.5}

Características neoliberales y propuestas de desarrollo regional equilibrado para el Plan Puebla Panamá.

\begin{tabular}{|l|l|}
\hline Características Neoliberal & Propuestas de Desarrollo Regional Equili- \\
\hline $\begin{array}{l}\text { Empresas disminuyen costos de } \\
\text { transporte por modernización de }\end{array}$ & $\begin{array}{l}\text { Campesinos disfrutan de carreteras que los une } \\
\text { a otras comunidades y a mercados }\end{array}$ \\
\hline Disminuye costos de mano de & La población tiene la opción de la capacitación \\
\hline Eliminan aranceles e impuestos & $\begin{array}{l}\text { Aceptación de la población con puesta en mar- } \\
\text { cha de alternativas de ingresos públicos para } \\
\text { no afectar el presupuesto y los programas so- } \\
\text { ciales y económicos. }\end{array}$ \\
\hline $\begin{array}{l}\text { Buscan vías de transporte rápidos } \\
\text { entre la región y el resto del mun- }\end{array}$ & $\begin{array}{l}\text { Aceptación de la población con información } \\
\text { sobre impactos y sus compensaciones. }\end{array}$ \\
\hline
\end{tabular}

E\&A IIES 


\begin{tabular}{|c|c|}
\hline Características Neoliberal & Propuestas de Desarrollo Regional Equili- \\
\hline $\begin{array}{l}\text { Acceso de empresas a principales } \\
\text { fuentes de energía } \\
\text { (hidroeléctricas) }\end{array}$ & $\begin{array}{l}\text { Aceptación de la población afectada y com- } \\
\text { pensaciones favorables por desubicación y da- } \\
\text { ños a ecosistemas }\end{array}$ \\
\hline $\begin{array}{l}\text { Propiedad privada de infraestruc- } \\
\text { tura de ferrocarril }\end{array}$ & $\begin{array}{l}\text { Informar de manera transparente y debatir los } \\
\text { beneficios hacia la población. }\end{array}$ \\
\hline $\begin{array}{l}\text { Propiedad privada de la biodiver- } \\
\text { sidad }\end{array}$ & $\begin{array}{l}\text { Exigir el cumplimiento de acuerdos internacio- } \\
\text { nal y del artículo } 169 \text { de la OIT sobre los dere- }\end{array}$ \\
\hline $\begin{array}{l}\text { Agronegocios de productos no } \\
\text { comestibles (eucalipto, teca, me- } \\
\text { lena, palma africana, etc.) }\end{array}$ & $\begin{array}{l}\text { Promover la productividad de comestibles para } \\
\text { la seguridad alimenticia como parte fundamen- } \\
\text { tal de la soberanía nacional. Ampliar el merca- } \\
\text { do doméstico de maíz, cereales, trigo, etc. }\end{array}$ \\
\hline $\begin{array}{l}\text { Impulsar el paso de mano de obra } \\
\text { campesina hacia la industria: ma- } \\
\text { quiladoras }\end{array}$ & $\begin{array}{l}\text { Impulsar programas de desarrollo endógeno } \\
\text { para aprovechar las capacidades y afianzar la } \\
\text { población rural y evitar la migración hacia la } \\
\text { ciudad con pérdidas culturales y problemas } \\
\text { urbanos. }\end{array}$ \\
\hline $\begin{array}{l}\text { Extracción de recursos estratégi- } \\
\text { cos nacionales (minerales, petró- } \\
\text { leo, agua y biodiversidad) }\end{array}$ & $\begin{array}{l}\text { La comunidad patentar el acervo cultural endó- } \\
\text { geno para generar ingresos locales. Preparar } \\
\text { recurso humano para ese fin. }\end{array}$ \\
\hline $\begin{array}{l}\text { Salida de campesinos hacia la } \\
\text { ciudad por falta de servicios en } \\
\text { zona rural. }\end{array}$ & $\begin{array}{l}\text { Papel del Estado para proveer servicios públi- } \\
\text { cos en zona rural y promover el empleo agríco- } \\
\text { la remunerado a través de aprovechar las po- } \\
\text { tencialidades endógenos. }\end{array}$ \\
\hline $\begin{array}{l}\text { Dependencia en inversiones ex- } \\
\text { tranjeras }\end{array}$ & $\begin{array}{l}\text { Autogestión para encontrar financiamiento } \\
\text { nacional e internacional. La búsqueda de infor- }\end{array}$ \\
\hline $\begin{array}{l}\text { El Estado deja en manos de la } \\
\text { iniciativa privada los servicios }\end{array}$ & $\begin{array}{l}\text { El Estado se asume como actor endógeno y } \\
\text { responsable del bienestar de sus representados. }\end{array}$ \\
\hline $\begin{array}{l}\text { Tecnología patentada por grandes } \\
\text { empresas extranjeras }\end{array}$ & $\begin{array}{l}\text { Tecnología apropiada endógena producto del } \\
\text { conocimiento y tradiciones comunales patenta- } \\
\text { das como propiedad intelectual comunitaria } \\
\text { derecho legítimo avalado por organizaciones } \\
\text { internacionales. }\end{array}$ \\
\hline $\begin{array}{l}\text { Desaparición de propiedad comu- } \\
\text { nal indígena y campesina de la } \\
\text { tierra }\end{array}$ & $\begin{array}{l}\text { Exigir el apego a los acuerdos internacionales } \\
\text { sobre el derecho indígena. Es además un dere- } \\
\text { cho humano. }\end{array}$ \\
\hline
\end{tabular}




\begin{tabular}{|l|l|}
\hline Características Neoliberal & Propuestas de Desarrollo Regional Equili- \\
\hline $\begin{array}{l}\text { Desaparición de la economía lo- } \\
\text { cal y los conocimientos locales }\end{array}$ & $\begin{array}{l}\text { Fortalecer el conocimiento local apoyado por } \\
\text { organismos internacionales y por las organiza- } \\
\text { ciones civiles que promueven la concientiza- } \\
\text { ción y el empoderamiento. }\end{array}$ \\
\hline $\begin{array}{l}\text { Desaparición de las concesiones } \\
\text { forestales comunitarias para dar } \\
\text { paso a nuevos latifundios empre- } \\
\text { sariales de monocultivo de expor- } \\
\text { tación. Patrimonio local pasa a }\end{array}$ & $\begin{array}{l}\text { El desarrollo endógeno como base para retener } \\
\text { a la población indígena y campesina en sus } \\
\text { tierras y gozar de sus derechos legítimos de los } \\
\text { recursos. Poner la situación a debate local, na- } \\
\text { cional e internacional. }\end{array}$ \\
\hline $\begin{array}{l}\text { Introducción de transgénicos, } \\
\text { contaminación genética de espe- } \\
\text { cies autóctonas. }\end{array}$ & $\begin{array}{l}\text { Movilizar a la población con concientización y } \\
\text { empoderamiento para la solidaridad regional } \\
\text { en protección de la biodiversidad autóctona. }\end{array}$ \\
\hline $\begin{array}{l}\text { Desaparición de la soberanía de } \\
\text { los países mesoamericanos }\end{array}$ & $\begin{array}{l}\text { El fortalecimiento de la democracia con la par- } \\
\text { ticipación ciudadana en la búsqueda de la auto- } \\
\text { nomía municipal y regional para promover el } \\
\text { desarrollo local y popular en reconocimiento } \\
\text { de los derechos indígenas. }\end{array}$ \\
\hline $\begin{array}{l}\text { Salud, cultura, educación necesa- } \\
\text { ria mínima para el desarrollo de } \\
\text { los habitantes, elemental para que } \\
\text { los individuos sean funcionales al } \\
\text { sistema. }\end{array}$ & $\begin{array}{l}\text { La salud, la cultura, la educación como estruc- } \\
\text { tura fundamental para la expansión de las liber- } \\
\text { tades individuales y para la transformación de } \\
\text { la sociedad que repercute en el aumento de la } \\
\text { productividad y competitividad. Desarrollo } \\
\text { endógeno que trasciende a integrarse a la glo- } \\
\text { balización. }\end{array}$ \\
\hline
\end{tabular}

Fuente: Elaboración propia principalmente en base al documento de la Associació d'Amistad amb el Poble de Guatemala, 2004.

Enfáticamente considero que las fuerzas para corregir el rumbo actual del PPP dependerán de la capacidad de los países miembros para: (i) fortalecer la organización de sus pueblos, (ii) buscar alternativas para la supervivencia y vida colectiva, (iii) abrir posibilidades factibles de producir, consumir, comercializar e invertir, (iv) emprender procesos de autogestión, movilización y auto transformación, ( $v$ ) promover la recuperación en colectivo del saber local de la gente y (vi) valorar la riqueza endógena. Sin embargo, coincido con Santana (1994: 203) en que "una de las mayores dificultades para la rápida ampliación y desarrollo de proyectos que involucren al tejido social, los municipios y las ONGs, radica en 
el poco estímulo que este tipo de proyectos recibe no sólo en los gobiernos locales sino regionales y nacional, como asimismo en la lentitud en el cambio de las políticas de las agencias de cooperación al desarrollo.

Junto al Estado garante de la protección de los individuos debe afirmarse progresivamente un Estado mediador del intercambio social y asociado a la sociedad civil (Perret, 1993: 314), pero el actor público ya no es sólo un mediador sino que un asociado activo; es quien reconoce y estimula la emergencia de actores colectivos salidos de la sociedad, y quien toma en cuenta sus iniciativas en la definición de las políticas sociales. En un mundo globalizado, donde las integraciones son una buena alternativa para impulsar el crecimiento y el desarrollo, el Estado tiene la oportunidad de ampliar su ámbito a esferas supranacionales. Este es el caso de la Unión Europa, donde un gobierno supranacional coordina la integración y la cooperación entre los países integrantes para el desarrollo regional buscando eliminar las desigualdades. El desarrollo de España, Portugal y Grecia son ejemplos de las prácticas de un Estado supranacional que ejerce la subsidiaridad, concepto que busca eliminar las desigualdades regionales.

La experiencia de la Unión Europea sirve de ejemplo para revisar las bases y lineamientos del papel del Estado en el PPP y para replantear las estrategias y las políticas públicas, mismas que le darían mayor legitimidad política y aportarían al desarrollo integral de las actividades económicas y al aspecto social. El papel del Estado en el PPP debe desplegarse en dos vertientes: un Estado que participe responsablemente a nivel microregional, que desde sus comunidades implemente políticas públicas endógenas y realice su gestión pública autóctonamente con características propias de su realidad microregional; y un Estado supranacional que se asuma como actor endógeno a la región mesoamericana enfocado a la subsidiaridad, implemente políticas públicas endógenas a la región y realice su gestión pública con características propias de su realidad regional.

Considero que la nueva gestión pública del PPP debe dar un salto cualitativo aprendiendo de los desaciertos que ha tenido la propuesta inicial y potencializando sus fortalezas endógenas. Los cambios hacia una NGP requieren de enmiendas constitucionales a favor del desarrollo endógeno. La calificada "aldea global" o la "comunidad planetaria" que justifica las integraciones como la iniciativa del PPP, debe conducir a igualdad en los niveles económicos, sociales y políticos entre países, en la expansión de las libertades de optar a mayor bienestar y al pleno respeto al empleo, a las culturas y a los recursos naturales. 
La NGP a nivel supranacional que requiere el PPP para el desarrollo equilibrado de Mesoamérica debe actuar con respeto hacia los habitantes y con responsabilidad en el desarrollo. Las propuestas, estrategias y políticas públicas que responden a factores exógenos deberán ponerse al debate público como sugiere Sen (2000) para que sea la libertad de las personas y de los grupos, ya sean sociales y culturales, para asumir los costos o los beneficios de su aplicación. Consecuentemente, si la modernización que propone el PPP lleva a la aceptación de los indígenas a renunciar a sus tierras, culturas y tradiciones y emigrar a las urbes, entonces tendremos que unirnos a Sachs (2006) por semejar la modernización con el desarrollo.

\section{PROPUSTA PARA EL DESARROLLO MESOAMERICANO EQUILIBRADO}

El planteamiento de políticas públicas endógenas para el desarrollo regional mesoamericano equilibrado es una alternativa para aminorar los efectos negativos de la globalización y para procurar una mayor distribución de los beneficios de la integración a nivel local y regional de los habitantes. La alternativa de políticas públicas de enfoque endógeno lleva a la responsabilidad del Estado en abrir los temas importantes de la propuesta de integración para el debate público para que la ciudadanía ejerza su libertad política de opinar. Esto se constituye en el ejercicio de derechos paralelos del grupo de interés de la población afectada, objeto de la integración. Estos derechos, que para el caso de Mesoamérica incluye a la de una población indígena y campesina, sirve como freno a la concentración del poder de decisión sólo entre el grupo de interés inversionista, empresarial y nacionales de los países desarrollados. Los indígenas y campesinos gozan de poder en el ámbito de sus economías locales, por su cultura, tradiciones y recursos que ostenta la tierra que les pertenece. Poder que tienen que valorar a través del fortalecimiento de la sociedad civil y las ONG que se dedican a concientizar y empoderar hacia esa valorización y que a su vez cuenta con apoyo de organizaciones internacionales y de acuerdos como el 169 de la OIT.

El debate que hoy enfrenta el capitalismo como modelo para generar desarrollo, ha llevado al surgimiento de nuevos paradigmas como el desarrollo endógeno y la nueva gestión pública para el desarrollo regional equilibrado. El siguiente cuadro nos contrasta las políticas públicas neoliberales y las endógenas del PPP.

El capitalismo ha justificado las desigualdades regionales, pero la política de desarrollo regional busca el desarrollo regional equilibrado. El liberalismo, sobre el cual se basa la ciencia económica y que impulsa la globalización es una amenaza a 
las economías locales. El proteccionismo es una defensa contra las crisis y una herramienta que puede permitir el desarrollo endógeno y local de sus habitantes, es parte del derecho humano para acceder a su desarrollo. Una vez fortalecidos en su desarrollo endógeno, los países podrían insertarse competitivamente a los mercados internacionales y a la globalización.

\section{Tabla No.6}

Políticas públicas versus el desarrollo endógeno en el marco del Plan Puebla Panamá.

\begin{tabular}{|c|c|}
\hline Neoliberales & Endógenas \\
\hline $\begin{array}{l}\text { De precios bajos y apertura comercial. } \\
\text { Resultado: Estrangula la mediana y peque- } \\
\text { ña economía campesina, destruyen la for- } \\
\text { ma de propiedad colectiva de la tierra. }\end{array}$ & $\begin{array}{l}\text { Para reforzar la capacidad organizati- } \\
\text { va para gestionar beneficios públicos } \\
\text { para el desarrollo endógeno y cumpli- } \\
\text { miento de acuerdos sobre derecho }\end{array}$ \\
\hline $\begin{array}{l}\text { De retiro de subsidios a servicios públicos } \\
\text { para mantener finanzas sanas. } \\
\text { Resultado: Manipulación de programas de } \\
\text { salud, educación, alimentación causa des- }\end{array}$ & $\begin{array}{l}\text { De búsqueda de equilibrio regional en } \\
\text { servicios sociales y aumento de ingre- } \\
\text { sos a través de promover la capacida- } \\
\text { des endógenas productivas del campo } \\
\text { para retener a la población. }\end{array}$ \\
\hline $\begin{array}{l}\text { De omisión de la transparencia y rendición } \\
\text { de cuentas. }\end{array}$ & $\begin{array}{l}\text { De nueva gestión pública que opera } \\
\text { con participación ciudadana, transpa- } \\
\text { rencia y rendición de cuentas. }\end{array}$ \\
\hline $\begin{array}{l}\text { De equilibrio presupuestal que lleva a eli- } \\
\text { minación de subsidios y a la privatización } \\
\text { de servicios sociales }\end{array}$ & $\begin{array}{l}\text { Sociales de educación, salud, vivien- } \\
\text { da, mercados, capacitación y de valo- } \\
\text { rización cultural. }\end{array}$ \\
\hline De inversión extranjera y empresas poten- & De inversión, MPyMES y promoción \\
\hline $\begin{array}{l}\text { De maquiladoras localizadas en centros } \\
\text { urbanos }\end{array}$ & $\begin{array}{l}\text { Migratorias para afianzar la poblacio- } \\
\text { nes en las zonas rurales y en las ciu- }\end{array}$ \\
\hline De privatización de la riqueza biodiversa & $\begin{array}{l}\text { Ambientales con participación de la } \\
\text { gente }\end{array}$ \\
\hline
\end{tabular}

Fuente: Elaboración propia principalmente en base al documento de la Associació d'Amistat amb el Poble de Guatemala, 2004. 
La resistencia social hacia el PPP a nivel ya prácticamente continental, es el reflejo de la demanda por la construcción de nuevos modelos de desarrollo, aunque aún falta una articulación y coherencia entre los diversos movimientos, todos ellos en su conjunto van dibujando bosquejos de una nueva sociedad, de nuevas formas de hacer política, nuevas visiones de desarrollo, ya no desde lo global ni sectorial, sino más bien desde lo local y lo integral pero con una articulación necesaria y sostenible con lo externo. La conjugación inteligente entre lo exógeno y lo endógeno. Según Sen (2000: 28), "con suficientes oportunidades sociales, los individuos pueden configurar en realidad su propio destino y ayudarse mutuamente. No tienen por qué concebirse como receptores pasivos de las prestaciones de ingeniosos programas de desarrollo". El debate público y la participación social son fundamentales para la elaboración de la política económica y social en un sistema democrático. La utilización de las prerrogativas democráticas -tanto de las libertades políticas como de los derechos humanosconstituye una parte crucial del ejercicio de la propia elaboración de la política económica, aparte de los demás papeles que puede tener.

La propuesta entonces consiste en no partir de una política homogénea en los procesos de integración regional, sino de una conjunción de visiones que orienten el desarrollo de los países integrantes en el corto y mediano plazos. Con este ejercicio tendríamos la posibilidad de incluir en el desarrollo agentes, territorios y procesos que a la fecha no han podido reconocer su lugar en la transformación y, por lo tanto, se han visto excluidos de los estímulos que los favorecen.

El punto de partida debe ser impulsar una nueva reingeniería de Estado que nos conduzca hacia un Estado democrático que centra su atención en la población, en sus capacidades y necesidades para llevar una vida digna. Un Estado responsable que maneje los recursos de su pueblo con respeto. Un Estado que sirva al pueblo, que busque con honradez la legitimidad de sus ciudadanos, que se asuma como servidor público. Para lograr esto, el Estado tiene que incorporar a la ciudadanía para reducir su margen de error, tomar la participación ciudadana como corresponsable en la gestión pública para concatenar los recursos económicos con los proyectos que impactarán en la vida y el trabajo de los habitantes en su globalidad. Al hacer a la ciudadanía coparticipe, necesariamente se trabaja con transparencia y rendición de cuentas. Hacer lo anterior nos posiciona en la práctica ante lo que teóricamente se conoce como la nueva gestión pública para el desarrollo regional equilibrado. La gestión pública no se puede copiar, porque no hay dos pueblos iguales, cada gestión pública debe ser autóctona. Hay que crear nuestras propias estructuras y políticas por país y luego por agrupación, en este 
caso, el PPP.

EI PPP no sólo requiere de la pertinencia de sus estrategias, sino también de una institucionalidad que permita llevarlas a cabo con eficiencia. Para este objetivo, se propone el establecimiento de una Gestión Pública Supranacional para el diseño de una estructura simplificada que permita generar una coordinación más eficiente entre las distintas áreas del PPP, a través de la conformación de consejos asesores en áreas claves para el desarrollo endógeno mesoamericano de Desarrollo Económico y Productividad, de Desarrollo Social, de Desarrollo Cultural y Patrimonio, entre otros, los cuales articularían los trabajos de las diversas iniciativas bajo un enfoque integral, y determinaría -de así requerirse- la creación de grupos de trabajo ad hoc para el desarrollo de programas y/o proyectos específicos a nivel microregional.

A nivel supranacional, los gobiernos de los países integrantes del PPP deben impulsar estrategias y políticas públicas ampliamente discutidas a nivel local en cada país, buscando el crecimiento conjunto y el desarrollo equitativo de la población mesoamericana. Plantear necesidades de infraestructura de acuerdo con sus necesidades de mejoramiento cuyos impactos sociales y ambientales sean mínimos. Gestionar financiamiento a nivel internacional con el aval de los estudios reales sobre los impactos. Como gobierno supranacional, buscar acompañamiento y asesoría de organismos internacionales que favorecen el desarrollo regional, la conservación del patrimonio cultural, el mejoramiento de la agricultura, el comercio justo, y en general, que promuevan la democracia y el buen gobierno.

A nivel microregional, el PPP debe enfocarse al desarrollo endógeno de su población campesina, indígena y rural. El Estado nacional debe proveer de escuelas, centros de salud, vivienda digna, apoyar la producción agrícola y artesanal articulándolos con los mercados urbanos, facilitar el crecimiento de microempresas comunitarias propiciando su encadenamiento. Estas estrategias generarían empleo que reducirían la emigración campo-ciudad. En los centros urbanos existen oportunidades de mejorar igualmente los servicios sociales, además de la generación de empleos bien pagados para dinamizar la economía doméstica.

El planteamiento de políticas públicas endógenas para el desarrollo regional mesoamericano equilibrado es una alternativa para aminorar los efectos negativos de la globalización y para procurar una mayor distribución de los beneficios de la integración a nivel local y regional de los habitantes. Buscando ese anhelado desarrollo mesoamericano equilibrado, el PPP debe tener un enfoque centrado en 
la gente y reforzar su derecho a ejercer su libertad desde el enfoque de Sen (2000). Así, no se estaría proponiendo un rompimiento total con los intereses exógenos dentro del cual se enmarca, dado que lo que se requiere es un complemento con los intereses endógenos tal como introdujo Yunus (2007) en Bangladesh a través de las empresas sociales.

La propuesta se basa en una toma de consciencia y en el fortalecimiento de las comunidades para que valoren sus recursos, sean estos humanos, culturales, ambientales y sociales, para establecer su inclusión como actores protagonistas en su propio ámbito como complemento, de igual importancia, de los intereses exógenos hacia ellos. Lo anterior es una manera de conllevar, de manera equilibrada, los intereses económicos, sociales, políticos y ambientales en beneficio de la población en general.

La alternativa de políticas públicas de enfoque endógeno lleva a la responsabilidad del Estado en abrir los temas importantes de la propuesta de integración al debate público para que la ciudadanía ejerza su libertad política de opinar. Esto se constituye en el ejercicio de derechos paralelos del grupo de interés de la población afectada, objeto de la integración. Estos derechos, que para el caso de Mesoamérica incluye a la de una población indígena y campesina, sirve como contrapeso a la concentración del poder de decisión sólo entre el grupo de interés inversionista, empresarial y nacionales de los países desarrollados. Los indígenas y campesinos gozan de poder en el ámbito de sus economías locales, por su cultura, tradiciones y recursos que ostentan. Poder que tienen que valorar a través del fortalecimiento de la sociedad civil y las ONG que se dedican a concientizar y empoderar hacia esa valorización y que a su vez, cuenta con apoyo de organizaciones internacionales y de acuerdos como el 169 de la OIT.

Abrir los temas del PPP a debate por la gente, es decir, transparentar y rendir cuentas e introducir la participación para corregir el rumbo de las políticas públicas, es encaminar al PPP en la dirección del desarrollo regional equilibrado de Mesoamérica con una nueva gestión pública. La participación política debe aumentar a través de la conformación de organizaciones regionales que vigilen situaciones claves de la región mesoamericana: organización indígena, organización campesina, organización ambiental, organización de productores, organización de consumidores con énfasis en lo endógeno de la región pero haciendo un frente común ante los modelos exógenos e incluso ante gobiernos que ignoran la importancia del modelo del desarrollo endógeno. 


\section{CONCLUSIONES}

La región sursureste de México comparte indicadores de marginación y de desarrollo humano similares a la región centroamericana lo cual se manifiesta a través del desequilibrio en el desarrollo regional y en la desigualdad de la distribución del ingreso. Lo anterior propicia la constitución de una región que busque conjuntamente la eliminación de los desequilibrios internos a sus países y entre los países de la región que se refleja en las diferencias de desarrollo y de nivel de ingresos entre, por ejemplo, Honduras, Costa Rica y Panamá. La iniciativa de integración debería enfatizar como patrimonio a sus poblaciones, las culturas, la biodiversidad y promoverlos en beneficio de sus habitantes.

En cuestión socioeconómica, se requiere la búsqueda de iniciativas que signifique avances significativos en el nivel de educación, salud, capacitación tecnológica e infraestructura de comunicaciones y transporte en beneficio de la productividad endógena.

Implementar un gobierno supranacional que conduzca a los países en lo individual y a la región hacia un desarrollo humano que se refleje en las superaciones en las trabas que hoy sufre el comercio de los productos de la región, la preparación del recurso humano y el desarrollo sustentable. El gobierno supranacional vigilaría los intereses de la región y crearía la sensibilidad de región integrada para buscar en común el crecimiento y el desarrollo bajo premisas de subsidiaridad, atrayendo proyectos y programas, así como financiamiento desde el exterior en apoyo del desarrollo endógeno y regional de Mesoamérica. El Plan Puebla Panamá requiere de esta reorientación, situación que no se ve mejorada con el proyecto Mesoamericano. La decisión que hay que tomar es continuar con iniciativas de integración que son exógenamente impuestas, o promover conjuntamente iniciativas que surjan desde las necesidades endógenas para el desarrollo endógeno y regional. Quién tiene la última palabra, los gobiernos de los países integrantes y sus habitantes, o los gobiernos promotores y la banca multinacionales? O se podrá llegar a un acuerdo entre todos los interesados? 


\section{REFERENCIAS}

Associació d'Amistat amb el Poble de Guatemala. (2004). El Plan Puebla Panamá.

Cuadernos de Guatemala. Número 1. Mayo de 2004.

Barreda, A. (2002). "El Plan Puebla Panamá". Biodiversidad No 33 Vol. 3. Agosto de 2002.

Bernard Perret y Guy Routang. (1993) La economía contra la sociedad. Crisis de la integración social y cultural. Fondo de Cultura Económica. Chile

Cazés, D. (coordinador). (1999). Creación de alternativas en México. Centro de Investigaciones Interdisciplinarias en Ciencias y Humanidades, Universidad Nacional Autónoma de México, México.

Chang, Ha-Joon. (1996). El papel del Estado en la economía. Colección ArielEconomía. Editorial Planeta. México.

Chambers, Robert. 1974. Managing Rural Development: Ideas and Experiences from East Africa. Uppsala. The Scandinavian Institute of Development Studies.

Centro de Investigación Económica y Política de Acción Comunitaria (CIEPAC), 2000. http.www.ciepac.mx. Consultado el 16 de febrero de 2011.

Chambers, Robert, et al. (editores). 1989. Farmers First: Farmers Innovation and Agricultural Research. London: Intermediate Technology Publications.

CONAPO (2005) Índice de Marginación, 2005. México. http.www.conapo.gob.mx. Consultado el 16 de febrero de 2011.

Dávila, Enrique, Georgina Kessel y Santiago Levy. (2004) “El sur también existe: un ensayo sobre el desarrollo regional de México (2000)" en Santiago Levy (Compilador), Ensayos sobre el Desarrollo Económico y Social en México. Fondo de Cultura Económica, México.

de Villalobos, Ruy. 2001. “Desarrollo Rural y Desarrollo Local: Descentralización y participación democrática como requisitos para una efectiva lucha contra la pobreza rural" en Burin y Heras (compiladores). Desarrollo Local: Una respuesta a escala humana a la globalización. Ediciones CICCUS- La Crujía, Argentina.

Fals Borda et.al. 1991. Acción y conocimiento. Como romper el monopolio con Investigación Acción Participativa. Bogota Columbia, CINEP. 
Freire, Paulo. 1970. The Pedagogy of the Oppressed. Herder and Herder. New York.

Gobierno de los Estados Unidos Mexicanos. (2000). Plan Puebla Panamá. Capítulo México. Documento base.

Gobierno de los Estados Unidos Mexicanos. (2001). Acuerdo por el que se crea la Coordinación General Plan Puebla-Panamá. D. O. F. 5 de junio de 2001

Gobierno de la República de El Salvador (2005) Diario Oficial. San Salvador, 9 de Marzo de 2005. Acuerdo de Sede entre la Comisión Ejecutiva del Plan Puebla Panamá y el Gobierno de la República de el Salvador.

Guerrero, Omar. (2003). Gerencia pública en la globalización. Universidad Autónoma del Estado de México, Facultad de Ciencias Políticas. Editorial Miguel Ángel Porrúa. México.

Jerez Henríquez, Bárbara. (s/f). La Resistencia al Plan Puebla Panamá en el istmo de Tehuantepec, México: construyendo un modelo de desarrollo desde las bases. Universidad Autónoma Chapingo, México.

Korten, David. 1980. Community organization and rural development: A learning process approach, Public Administration Review. September - October, 1980. pp $480-510$.

Korten, David y R. Klass. 1984. People centered Development: Contributions Towards Theory and Planning development. West Hartford, Kumarian Press.

Kretzmann, J.P. y McKnight, J.L. 1993. Building Communities from the Inside Out. A Path Towards Finding And Mobilizing a Community's Assets. ACTA Publications, Chicago, Illinois, USA.

Licha, Isabel. 2002. "El debate actual sobre desarrollo participativo" en Licha, Isabel (editora). Gerencia Social en América Latina: Enfoque y experiencias innovadoras. Banco Interamericano de Desarrollo, Washington, D.C.

Ornelas Delgado, Jaime. (2004). “El Plan Puebla-Panamá y la globalización neoliberal"

Aportes: Revista de la Facultad de Economía-BUAP. Año VII, Núm. 21.

Perret, B. y Guy Routang. (1993). La economía contra la sociedad. Crisis de la integración social y cultural. Fondo de Cultura Económica. Chile. 
Programas de las Américas. (2002). Resistencia Ciudadana al Plan Puebla Panamá. Interhemispheric Resource Center. Serie Acción Ciudadana de las Américas. No. 2. Septiembre 2002.

Rodríguez, Nemensio J. (2009). “Boceto para Guerrero del Plan Puebla Panamá: rediseño espacial para una globalización a la medida" en Programa Universitario México, nación multicultural - UNAM y la Secretaría de Asuntos Indígenas del Gobierno del estado de Guerrero, Estado de Desarrollo Económico y Social de los pueblos indígenas de Guerrero. México.

Roldan Dávila, Genoveva (2001). "Globalización y derecho al desarrollo como derecho humano", en Estay, Jaime; Girón, Alicia y Martínez, Osvaldo (Coordinadores). Globalización de la economía mundial. Principales dimensiones en el umbral del siglo XXI. Colección Jesús Silva Herzog. Universidad Nacional Autónoma de México, Instituto de Investigaciones Económicas. Editorial Miguel Ángel Purrúa. México.

Sachs, Ignacy. 1976. The Discovery of the Third World. Cambridge, Ma. MIT Press.

Sachs, J. (2006). El fin de la pobreza, Cómo conseguirlo en nuestro tiempo. Editorial Arena Abierta. México.

Santana Rodríguez, Pedro. (1994). "Gobiernos locales, descentralización y democracia en Colombia" en Nuevas Políticas Urbanas. Las ONG y los gobiernos municipales en la democratización latinoamericana. Charles A. Reilly (Compilador). Fundación Interamericana, Arlington, Virginia, E. U.

Schumacher, E. F. 1973. Small is Beautiful: Economics as if People Mattered. New York, Perenial Library and London, Abacus.

Secretaría de Educación Pública. 1967. Investigación, Organización y Desarrollo de la Comunidad, Segunda edición, Ediciones Oasis, Monterrey, México.

Sen, Amartya (2000). Desarrollo y Libertad. Editorial Planeta. México.

Slocum y Thomas. 2001. "Participation, empowerment and sustainable development" en Slocum, Wichhart, et al., (editors). Power, Process and Participation: Tools for Change. ITDG Publishing, London, UK.

Tamames, Ramón. (1991) Estructura económica internacional. Editorial Alianza y el Consejo Nacional par alas Culturas y las Artes. México.

United Nation New York. USA. 
United Nation New York. USA.

Yunus, M. (2007). Creating a world without poverty. Social business and the future of capitalism. Public Affairs, New York. USA. 